\title{
ANÁLISE DA COBERTURA VACINAL CONTRA INFLUENZA NO ESTADO DE MINAS GERAIS
}

\author{
ANALYSIS OF VACCINE COVERAGE AGAINST INFLUENZA \\ IN THE STATE OF MINAS GERAIS
}

\section{ANÁLISIS DE LA COBERTURA DE VACUNA CONTRA INFLUENZA EN EL ESTADO DE MINAS GERAIS}

\author{
Tarcísio Laerte Gontijo * \\ Valéria CONCEIÇÃo de Oliveira ** \\ Nívea Aparecida de Almeida ${ }^{* * *}$ \\ Eliete Albano de Azevedo Guimarães ${ }^{* * *}$
}

\begin{abstract}
RESUMO
Objetivo: Analisar a cobertura vacinal contra influenza de idosos, gestantes e crianças nos 853 municípios de Minas Gerais. Método: Estudo ecológico que teve como unidades de análise os municípios do estado de Minas Gerais. Foram calculadas as coberturas vacinais contra influenza, utilizando-se a base de dados do Programa Nacional de Imunização. Os dados foram analisados no software estatístico SPSS ${ }^{\circledR} 17.0$ por meio de análise descritiva e testes de associação. Resultados: O estado de Minas Gerais alcançou a meta mínima de cobertura para os três grupos estudados, porém, a análise por município mostrou que 316 (32\%) deles não atingiram a meta para gestante, 17 (2\%) para crianças e $41(4,8 \%)$ para idosos. Associações das variáveis de contexto (cobertura da estratégia de saúde da família e porte populacional) com o alcance da meta mínima da cobertura vacinal não foram evidenciadas. Conclusão: Minas Gerais atingiu a cobertura vacinal contra influenza, contudo percebe-se baixa homogeneidade da cobertura no grupo gestante e crianças.
\end{abstract}

Palavras chave: Vacinas contra influenza, cobertura vacinal, avaliação em saúde, enfermagem em saúde comunitária.

\begin{abstract}
Objective: Analyze influenza vaccination coverage of the elderly, pregnant women and children in 853 municipalities of Minas Gerais in 2013. Method: Ecological study, the unit of analysis was immunization coverage in the municipalities. The dependent variable was influenza vaccination coverage of priority groups (children between six months and two years, the elderly and pregnant women). The independent variables were: population size and family health strategy covering. Data were analyzed through the statistical program SPSS 17.0 by means of descriptive association and analysis tests. Results: In the analysis of vaccination coverage by municipality low homogeneity in the pregnant and children groups was found. 316 (32\%) did

\footnotetext{
* Doutor. Professor do curso de Enfermagem da Universidade Federal de São João del-Rei, Divinópolis, Minas Gerais, Brasil. Email: enftarcisio@ufsj.edu.br. Autor correspondente.

${ }^{* *}$ Doutor. Professor do curso de Enfermagem da Universidade Federal de São João del-Rei, Divinópolis, Minas Gerais, Brasil. Email: valeria.oli.enf@gmail.com

${ }^{* * *}$ Enfermeira. Universidade Federal de São João del-Rei. Divinópolis, Minas Gerais, Brasil. Email: nívea.almeida@hotmail.com ${ }^{* * * *}$ Doutor. Professor do curso de Enfermagem da Universidade Federal de São João del-Rei, Divinópolis, Minas Gerais, Brasil.Email: elietealbano@ufsj.edu.br
} 
not have coverage for pregnant women, 17 (2\%) for children and $41(4.8 \%)$ for the elderly. Associations of context variables (coverage strategy of family health and population size) to reach minimum coverage were not identified. Conclusion: Although the state has appropriate vaccine coverage, coverage homogeneity for pregnant women and children remains low.

Key words: Influenza vaccines, immunization coverage, health evaluation, community health nursing.

\section{RESUMEN}

Objetivo: Analizar la cobertura de vacunación contra la gripe de los ancianos, las mujeres embarazadas y los niños en los 853 municipios de Minas Gerais. Material y método: Estudio ecológico que tuvo como unidades de análisis los municipios del estado de Minas Gerais. Los datos fueron analizados mediante el programa estadístico SPSS $17.0^{\circledR}$ a través de pruebas de análisis y asociación descriptivos. Resultados: El estado de Minas Gerais alcanzó la cobertura mínima para los tres grupos, sin embargo, el análisis por municipios mostró que 316 (32\%) no alcanzó la cobertura para las mujeres embarazadas, 17 (2\%) niños y 41 (4,8\%) para los ancianos. No se identificó asociaciones de variables de contexto (estrategia de cobertura de salud de la familia y tamaño de la población) para alcanzar la cobertura mínima. Conclusión: Aunque el estado funcione bien, se percibe bajo la homogeneidad de la cobertura en el grupo de embarazadas y niños.

Palabras clave: Vacunas contra la influenza, cobertura de vacunación, evaluación en salud, enfermería en salud comunitaria.

Fecha recepción: 02/09/15 Fecha aceptación: 02/08/17

\section{INTRODUÇÃO}

O vírus da influenza é um dos principais responsáveis pelas infecções respiratórias agudas, atingindo principalmente crianças menores de dois anos de idade, gestantes e idosos. Anualmente cerca de $10 \%$ da população mundial é acometida por essa infecção, que tem elevada transmissibilidade e estimase que ocorram mais de 250 mil óbitos em todo o mundo (1). A infecção pelo influenza e suas complicações são responsáveis pelo aumento do risco de morbimortalidade nos grupos vulneráveis (2).

Para uma prevenção efetiva desse agravo é necessária vigilância qualificada e vacinação, que é considerada uma das intervenções mais custo-efetivas e seguras (3-5). A vacina contra influenza apresenta eficácia geral de $67 \%$, sendo os adultos e crianças os grupos mais beneficiados (6).

No Brasil, o Ministério da Saúde (MS) realiza, desde 1999, campanhas anuais de vacinação contra influenza, aos grupos mais susceptíveis com o objetivo de reduzir a mortalidade, as complicações e as internações decorrentes dessa infecção (3). Inicialmente a vacina foi disponibiliza apenas para idosos, contudo no ano de 2010 a vacinação foi estendida às crianças e gestantes.

Atualmente os grupos prioritários recomendados pela Organização Mundial de Saúde (OMS) e incluídos na estratégia do MS do Brasil, são pessoas com 60 anos ou mais, gestantes, mulheres em puerpério, crianças entre seis meses e 5 anos de idade, profissionais de saúde, indígenas, pessoas privados de liberdade, além dos doentes crônicos e transplantados (3).

Identifica-se forte impacto da vacinação contra influenza na redução de internações e mortes por doenças respiratórias em diferentes partes do mundo (7). Entretanto, estudos realizados no Brasil mostram que a vacina, mesmo sendo gratuita, ainda não atinge co- 
berturas adequadas, alcançando baixas coberturas $(8,9)$.

A manutenção de altas coberturas vacinais é indispensável para uma efetiva proteção da população, principalmente dos grupos mais susceptíveis. Coberturas vacinais elevadas e homogêneas nos diferentes municípios constituem um bom indicador de saúde pública (5). A cobertura vacinal alcançada por um estado ou país, mesmo sendo elevada no geral, pode não ser homogênea entre as diferentes cidades. Em países e estados que possuem grande número de cidades, como no Brasil, pode haver regiões com coberturas mais baixas que o geral, aumentando assim o risco de adoecimento dessas populações específicas. Por isso, torna-se importante realizar análises de cobertura vacinal por municípios, permitindo assim verificar a existência de áreas com problemas vacinais (8).

No contexto do serviço público de saúde brasileiro a enfermagem é a principal responsável pelas atividades de imunização. Sendo assim, é importante que a mesma tenha conhecimento da cobertura vacinal e busque compreender as variações verificadas nesse indicador e os possíveis fatores determinantes para o não alcance das coberturas preconizadas.

Assim, este estudo teve como objetivo analisar a cobertura vacinal contra influenza de idosos, gestantes e crianças menores de dois anos nos 853 municípios do estado de Minas Gerais.

\section{MÉTODO}

Trata-se de um estudo ecológico realizado no estado de Minas Gerais que é composto por 853 municípios e está divido em 13 regiões ampliadas de saúde. Sua população é estimada em 19,8 milhões de habitantes, sendo cerca de $10 \%$ maiores de 60 anos de idade (10).

Este estudo teve como unidades de análise os 853 municípios de Minas Gerais. A esco- lha por este estado se deu devido ao fato dele possuir o maior número de municípios da federação. A variável dependente foi a cobertura vacinal contra influenza de três grupos prioritários (crianças entre seis meses e dois anos de idade, pessoas acima de 60 anos e gestantes), de cada município. Vale ressaltar que a campanha nacional de vacinação contra influenza, no Brasil, estabelece ainda outros grupos populacionais como prioritários, porém por condições técnicas e operacionais este estudo optou por incluir na análise as coberturas dos três grupos citados acima.

As variáveis independentes, consideradas de contexto, foram o porte populacional e a cobertura de estratégia de saúde da família (ESF) do mesmo ano. Os dados relativos à cobertura vacinal, referentes ao ano 2013, foram obtidos por meio da base de dados do Programa Nacional de Imunização (PNI), disponível no Departamento de Informática do SUS (DATASUS). Obteve-se a cobertura de ESF nas bases de dados da Secretaria Estadual de Saúde de Minas Gerais e o porte populacional nas bases de dados do Instituto Brasileiro de Geografia e Estatística (IBGE).

Realiza-se o processamento e análise dos dados no software Statistical Package for the Social Sciences (SPSS ${ }^{\circledR}$ ) for Windows versão 17.0. Inicialmente realiza-se análise descritiva de todas variáveis estudadas e calcula-se as coberturas vacinais de cada município. A partir dessa análise os municípios foram estratificados entre aqueles que alcançaram a cobertura mínima de $80 \%$ e os que não alcançaram em cada um dos grupos. Para avaliar possíveis fatores associados ao alcance da cobertura mínima de $80 \%$ realizase análise univariada por meio do teste de qui-quadrado de Pearson, fixando um nível de significância de 5\%. Foi ainda estimado a força de associação calculando a razão de chances (odds ratio) por meio do teste de Mantel-Haenszel com seus respectivos intervalos de confiança de 95\%.

Este estudo obedece aos princípios éticos da Resolução 466/12 do Conselho Nacional 
de Saúde e os dados utilizados foram acessados em bancos de dados oficiais e de acesso livres o que justifica a ausência do parecer de Comitê de Ética em Pesquisa.

\section{RESULTADOS}

A população estimada a ser vacinada contra o vírus influenza, considerando todos os grupos prioritários, no estado de Minas Gerais foi de 3.278.748 habitantes. Foram vacinados 2.973.696 habitantes, atingindo uma cobertura vacinal total de $90,7 \%$. A cobertura vacinal alcançada em cada um dos grupos estudados foi superior a $80 \%$, meta mínima preconizada pelo MS. Destaca-se alta cobertura entre as crianças de seis meses e dois anos de idade $(99,49 \%)$ (Tabela 1$)$.

$\mathrm{Na}$ análise da cobertura vacinal percebese baixa homogeneidade no grupo gestante e crianças (Tabela 2). Identifica-se que 316 $(37,0 \%)$ dos municípios mineiros não atingiram a meta mínima preconizada no grupo de gestantes. Já no grupo crianças e idosos, 17
(2,0\%) e 41 (4,8\%) dos municípios não atingiram a cobertura mínima, respectivamente. Outro ponto importante é o percentual de municípios com coberturas acima de 100\%. Destaca-se ainda que 28 (3,3\%) municípios registraram cobertura vacinal para crianças de exatamente $100 \%$, o mesmo acontecendo para 25 (2,9\%) municípios no grupo gestante.

A Tabela 3 mostra a análise descritiva da cobertura vacinal para os grupos prioritários. Nota-se um desvio padrão alto quando analisado a cobertura vacinal das gestantes e crianças, indicando uma alta dispersão dos escores em torno da média, contendo valores extremos entre coberturas mínimas e máximas. Esses achados reforçam a idéia de baixa homogeneidade entre as coberturas dos diferentes municípios analisados.

Não identifica-se associações das variáveis de contexto estudadas, cobertura da estratégia de saúde da família e o porte populacional do município com o alcance da cobertura vacinal nos três grupos prioritários estudados (Tabela 4).

Tabela 1. População estimada, doses aplicadas e cobertura vacinal contra influenza de grupos prioritários, Minas Gerais, 2013.

\begin{tabular}{lccc}
\hline Grupo & População estimada & Doses aplicadas & Cobertura (\%) \\
\hline Idosos & 2.337 .624 & 2.049 .022 & 87,7 \\
Gestantes & 195.016 & 161.055 & 82,6 \\
Crianças & 390.040 & 388.032 & 99,5 \\
\hline
\end{tabular}

Fonte: Sistema de informação nominal do Programa Nacional de Imunizações (SI-PNI).

Tabela 2. Distribuição da cobertura vacinal contra influenza segundo os grupos prioritários, Minas Gerais, 2013.

\begin{tabular}{lcccccc}
\hline \multirow{2}{*}{ Grupos } & \multicolumn{9}{c}{ Cobertura Vacinal (\%) } & \multicolumn{2}{c}{ Maior 100\% } \\
\cline { 2 - 7 } & $\mathrm{n}$ & $\%$ & $\mathrm{n}$ & $\%$ & $\mathrm{n}$ & $\%$ \\
\hline Idosos & 41 & 4,8 & 690 & 80,3 & 122 & 14,9 \\
Gestantes & 316 & 37 & 368 & 43,2 & 169 & 19,8 \\
Crianças & 17 & 2 & 391 & 45,9 & 444 & 52,1 \\
\hline
\end{tabular}


Tabela 3. Análise descritiva da cobertura vacinal, Minas Gerais, 2013.

\begin{tabular}{lcccc}
\hline \multirow{2}{*}{ Grupos prioritários } & \multicolumn{4}{c}{ Cobertura vacinal (\%) } \\
\cline { 2 - 5 } & Mínima & Média & Máxima & Desvio Padrão \\
\hline Crianças & 57,8 & 104,7 & 327,8 & 20,5 \\
Gestantes & 1,4 & 86,3 & 388,9 & 24,4 \\
Idosos & 16,6 & 90,8 & 147,2 & 9,3 \\
\hline
\end{tabular}

Tabela 4. Associação de variáveis relacionadas com o alcance da meta mínima de cobertura vacinal contra influenza nos grupos prioritários, Minas Gerais, 2013.

\begin{tabular}{lcccccc}
\hline & \multicolumn{5}{c}{ Cobertura vacinal } \\
\cline { 2 - 6 } Variáveis & \multicolumn{2}{c}{ Crianças } & \multicolumn{2}{c}{ Gestantes } & \multicolumn{2}{c}{ Idosos } \\
\cline { 2 - 7 } & $\mathrm{OR}^{\star}$ & $\mathrm{p}$ valor & $\mathrm{OR}^{*}$ & $\mathrm{p}$ valor & $\mathrm{OR}^{*}$ & $\mathrm{p}$ valor \\
\hline $\begin{array}{l}\text { Cobertura de Saúde da Família } \\
\quad \text { Menor que 70\% }\end{array}$ & 1 & & 1 & & 1 & \\
$\quad$ 70\% e mais & 0,25 & 0,181 & 0,79 & 0,198 & 1,74 & 0,118 \\
Número de habitantes & & & & & & \\
$\quad$ Menos de 10 mil & 1 & & 1 & & 1 & \\
$\quad 10$ mil e mais & 0,91 & 0,846 & 1,14 & 0,348 & 1,39 & 0,308 \\
\hline
\end{tabular}

* Odds Ratio obtido por meio do teste Mantel-Haenszel.

\section{DISCUSSÃO E CONCLUSÃO}

Minas Gerais é o estado brasileiro com o maior número de municípios do país, fazendo com que o alcance de coberturas vacinais desejáveis e homogêneas em todas as cidades seja uma tarefa complexa. Historicamente, Minas Gerais tem atingido a meta mínima de cobertura vacinal tanto para a população de crianças quanto de idosos. A cobertura de idosos vem apresentando crescimento variando de 75,6\% em 2007 a 87,7\% em 2013. Porém o mesmo não acontece com gestantes que apresentam cobertura vacinal sem um padrão de crescimento nos últimos quatro $\operatorname{anos}(3)$.

Os resultados mostraram que as maiores dificuldades para alcance da cobertura foram no grupo de gestantes. Os motivos de não vacinação da gestante estão relacionados a fatores pessoais, familiares e culturais, fatores socioeconômicos desfavoráveis que dificultam o acesso ao serviço de saúde, fatores institucionais, como a alta rotatividade dos profissionais da saúde, vínculo entre o binômio gestante e equipe de saúde diminuída, consultas de pré-natal inadequadas, falta de material e infraestrutura, entre outros (11). Estudos apontam também que além de aspectos clínicos e comportamentais, o medo, à falta de informação aos mitos e crenças que a população traz nas suas representações e nos seus valores culturais também estão envolvidos a não adesão na vacinação de gestantes $(12,13)$.

Além disso, outra pesquisa com o objetivo de compreender os motivos que levaram as gestantes a não se vacinarem contra a influenza observou a falta de orientação acerca da vacina durante a realização do pré-natal, reforçando a necessidade do desenvolvimen- 
to de estratégias de comunicação entre o profissional de saúde e as gestantes (11).

Gestantes com melhor grau de informação sobre os efeitos protetores da vacinação contra a gripe materna tiveram melhor aceitação da vacina. Em geral, as mulheres grávidas vacinadas tiveram um maior conhecimento e atitudes mais positivas em relação a vacinação da gripe do que as mulheres grávidas não vacinadas, além de confiar na segurança e eficácia da vacina (14). Por outro lado, os autores referidos relataram que mulheres grávidas com co-morbidades tiveram maiores probabilidades de serem vacinadas. Gestantes que não aceitaram a vacina declararam bom estado de saúde e imunidade pré-existente, considerando a vacinação desnecessária. E ainda, essas gestantes classificaram a gripe como uma doença não muito grave ou se infectadas, a doença não iria fazê-las muito doente. Algumas participantes do estudo preferiam confiar em tratamento medicamentoso para curar a gripe, se necessário, do que se vacinarem (14).

Outro ponto encontrado nos resultados foi o número de cidades com coberturas superiores a $100 \%$, sendo que, coincidentemente um grande número destas cidades alcançou coberturas de exatamente 100\%. Pressupõe-se que coberturas vacinais superiores a $100 \%$ pode ser explicado por erros de registro dos instrumentos de doses aplicadas, como a duplicidade de dados, principalmente nas doses aplicadas nas crianças menores de nove anos ao receberem a vacina pela primeira vez, as quais requerem duas doses com intervalo de 4 a 6 semanas. Outro pressuposto refere-se a população alvo utilizada para medir a cobertura vacinal. Geralmente as populações são estimativas do Instituto Brasileiro de Geografia e Estatística que utiliza dados dos dois últimos censos. Entretanto, em algumas regiões as mudanças no crescimento populacional são muito rápidas, consequentemente não sendo captadas pelos métodos de estimativas empregados (15).

Há disponível na literatura estudos que apontam fatores associados ao comportamento dos indivíduos (idosos, crianças e gestantes) frente a vacinação. Por outro lado, há certa escassez de estudos que mostram fatores associados ao alcance das coberturas relacionados com o contexto e organização das campanhas. Os resultados deste estudo mostram que o porte populacional do município e a cobertura de ESF não tiveram influência no resultado da campanha. Esperava-se que coberturas elevadas da ESF e municípios de menor porte apresentassem resultados melhores, uma vez que é possível ter um controle mais preciso sobre a população a ser vacinada.

Destacam-se como limitações deste estudo, a utilização de dados secundários, o que pode comprometer a qualidade da informação e o tipo de delineamento ecológico, que não permite inferências para o nível individual (falácia ecológica). Contudo, este estudo fornece dados importantes para a enfermagem, que atua diretamente na sala de vacina, sobre os grupos vulneráveis, porém resistentes à vacinação. E aponta a necessidade de estabelecer estratégias de vigilância que garantam a adesão à campanha contra influenza, principalmente no grupo de crianças e gestantes.

Ademais, os resultados deste estudo permitem concluir que o estado de Minas Gerais atingiu a cobertura vacinal contra influenza, porém percebe-se baixa homogeneidade da cobertura no grupo gestante e crianças. Mesmo havendo sucesso na campanha vacinal é necessário compreender melhor os fatores que expliquem baixas coberturas em alguns municípios para auxiliá-los no alcance de suas metas. É importante ainda destacar que devem ser realizadas ações de sensibilização das gestantes que ainda se mostram resistentes a vacinação contra influenza.

Sendo assim é importante que se tenha conhecimento sobre a vacinação da gestante, e que se invista na orientação e seguimento dos profissionais que atendem essas mulheres, promovendo o conhecimento, pois ele 
constitui o elemento essencial para a continuidade e o sucesso dos programas de imunização.

\section{REFERÊNCIAS}

1. Kfouri RA, Richtmann R. Influenza vaccine in pregnant women: immunization coverage and associated factors. Einstein (Sao Paulo). 2013; 11(1): 53-7.

2. Daufenbach LZ, Duarte EC, Carmo EH, Campagna AS, Santos CAST. The impact of vaccination on influenza-related hospitalizations of the elderly in Brazil. Epidemiol Serv Saude. 2014; 23(1): 9-14.

3. Ministério da Saúde (BR). Informe técnico: 19a Campanha Nacional de Vacinação Contra a Influenza. Brasília: Ministério da Saúde; 2017.

4. Luna EJA, Gattás VL, Campos SRSLC. Effectiveness of the Brazilian influenza vaccination policy: a systematic review. Epidemiol Serv Saude. 2014; 23(3): 55975.

5. Luhm KR, Cardoso MRA, Waldman EA. Cobertura vacinal em menores de dois anos a partir de registro informatizado de imunização em Curitiba, PR. Rev Saude Publica. 2011; 45(1): 90-8.

6. Osterholm MT, Kelley NS, Sommer A, Belongia EA. Efficacy and effectiveness of influenza vaccines: a systematic review and meta-analysis. Lancet Infect Dis. 2012; 12(1): 36-44.

7. Bós AJG, Mirandola AR. Cobertura vacinal está relacionada à menor mortalidade por doenças respiratórias. Cien Saude Colet. 2013; 18(5): 1459-62.

8. Moura RF, Andrade FB, Duarte YAO, Lebrão ML, Antunes JLF. Factores relacionados con la adhesión a la vacunación antigripal en ancianos que viven en la comunidad, São Paulo, Brasil. Cad Saude Publica. 2015; 31(10): 2157-68.

9. Francisco PM, Donalisio MR, Barros MB, César CL, Carandina L, Goldbaum M. Fatores associados à vacinação contra a influenza em idosos. Rev Panam Salud Publica. 2006; 19(4): 259-64.

10. Estimativas de população [Internet]. Rio de Janeiro (RJ): Instituto Brasileiro de Geografia e Estatística; 2014 [citado 17 mar 2014]. Disponível em: ftp://ftp.ibge. gov.br/Estimativas_de_Populacao/Estimativas_2013/estimativa_2013_dou.pdf

11. Pereira BFB, Martins MA, Barbosa TLA, Silva CSO, Gomes LMX. Motivos que levaram as gestantes a não se vacinarem contra H1N1. Cien Saude Colet. 2013; 18(6): 1745-1752.

12. Nascimento EFA, Faria AL, Nakamiti MCP, Santos TCMM, Nunes NAH. Cobertura vacinal dos idosos de um grupo de convivência da Universidade de Taubaté, São Paulo, Brasil. J Nurs UFPE on line [Internet]. 2009 Jul [citado 25 mai 2015]; 3(3): 520-3. Disponível em: https://periodicos.ufpe.br/revistas/revistaenfermagem/article/view/5631

13. Victor JF, Gomes GD, Sarmento LR, Soares AMG, Mota FRN, Leite BMB, Soares ES, Silva MJ. Factors associated with vaccination against influenza a (H1N1) in the elderly. Rev Esc Enferm USP. 2014; 48(1): 58-65.

14. Yuen CYS, Tarrant M. Determinants of uptake of influenza vaccination among pregnant women-A systematic review. Vaccine. 2014; 32(36): 4602-4613.

15. Moraes JC, Ribeiro MCSA, Simões O, PC, Barata RB. Qual é a cobertura vacinal real? Epidemiol Serv Saude [Internet]. 2003 Set [citado 17 abr 2015]; 12(3): 147-153. Disponível em: http://dx.doi. org/10.5123/S1679-49742003000300005 